\title{
5G New Radio Requires the Best Possible Risk Assessment Studies: Perspective and Recommended Guidelines
}

\author{
Mats-Olof Mattsson ${ }^{1,2 t}$, Myrtill Simkó ${ }^{1,2 * t}$ and Kenneth R. Foster ${ }^{3 t}$ \\ ${ }^{1}$ SciProof International AB, Östersund, Sweden, ${ }^{2}$ Institute for Advanced Studies, Strömstad Academy, Strömstad, Sweden, \\ ${ }^{3}$ Department of Bioengineering, University of Pennsylvania, Philadelphia, PA, United States
}

\section{OPEN ACCESS}

Edited by:

Luca Chiaraviglio,

University of Rome Tor Vergata, Italy

Reviewed by:

Alexander Lerchl,

Jacobs University Bremen, Germany

Ahmed Elzanaty,

King Abdullah University of Science

and Technology, Saudi Arabia

*Correspondence: Myrtill Simkó myrtill.simko@sciproofinternational.se

${ }^{t}$ These authors have contributed equally to this work

Specialty section:

This article was submitted to

Wireless Communications,

a section of the journal

Frontiers in Communications and

Networks

Received: 14 June 2021 Accepted: 03 September 2021 Published: 22 September 2021

Citation:

Mattsson M-O Simkó M and Foster KR (2021) 5G New Radio Requires the

Best Possible Risk Assessment

Studies: Perspective and

Recommended Guidelines.

Front. Comms. Net 2:724772.

doi: 10.3389/frcmn.2021.724772
The development and establishment of mobile communication technologies has necessitated assessments of possible risks to human health from exposures to radiofrequency electromagnetic fields (RF EMF). A number of expert committees have concluded that there is no evidence for such risks as long as exposures are at or below levels that do not allow tissue heating. These assessments have been based primarily on studies investigating frequencies up to $6 \mathrm{GHz}$ including frequencies similar to those used by two of three major bands of fifth generation (more accurately $5 \mathrm{G}$ New Radio or $5 \mathrm{G} \mathrm{NR}$ ) of mobile communication. Bioeffects studies in so-called high-band at 25-39 GHz are particularly sparse. Future assessments relevant for these frequencies will need to rely on still unperformed studies. Due to few available studies at 5G NR "high band" frequencies, and questions raised by some existing studies, a recent review recommended a wide range of RF biostudies be done at $5 G$ NR "high band" frequencies. It is of importance that such studies be done using the best possible science. Here we suggest factors to consider when performing future studies in this area. The present focus is on laboratory studies to clarify biological effects of radiofrequency (RF) energy at $5 \mathrm{G}$ "high band" frequencies and, more generally at millimeter wave (mm-wave) frequencies $(30-300 \mathrm{GHz})$ which will be increasingly used by communications technologies in the future. Similar comments would apply to epidemiology and exposure assessment studies, but those are not the focus of the present Perspective.

Keywords: 5G, risk assessment, RF EMF, mobile phone, risk of bias

\section{INTRODUCTION}

Numerous studies have investigated possible biological and health-related effects of radio frequency electromagnetic fields (RF EMF) that are used for mobile communication (1G-4G mobile telephony). Both national and international expert committees with mandates from public institutions have assessed published health effect studies related to such RF EMF exposures. The committees mainly reached the same conclusion, viz. that exposures at or below exposure guidelines provided by the International Committee on Non-Ionising Radiation (ICNIRP) (International Commission on Non-Ionizing Radiation Protection (ICNIRP)) or IEEE-International Commission on Electromagnetic Safety (IEEE-ICES) (IEEE, 2019) pose no identifiable health risks for the exposed population. 
“5G” (more accurately 5G New Radio or 5G NR) refers to a "fifth generation" standard (set of specifications) that allow cellular networks to communicate. The 5G standard is presently defined for three frequency bands, which vary somewhat in different countries. "Low-band" $5 \mathrm{G}$ operates at $600-700 \mathrm{MHz}$, similar to that used by some present-day cellular networks. Mid-band $5 \mathrm{G}$ operates in a somewhat higher range, $2.5-3.7 \mathrm{GHz}$, which is close to a frequency range used by some $4 \mathrm{G}$ networks $(2.5 \mathrm{GHz})$. High-band $5 \mathrm{G}$ (25-39 GHz) operates near the lower end of the millimeter wave band (which extends between 30 and $300 \mathrm{GHz}$ ). To the extent that the RF energy emitted from $5 \mathrm{G}$ systems has distinctive risk issues, they are related to high-band systems for which safety of the RF exposure is much less well studied than for RF signals at lower frequencies.

For assessment of any possible effects occurring at these presently little used (by the public) frequencies, assessors will rely on studies that are specifically investigating these frequencies. Presently, the number of available bioeffects studies at high-band 5G NR frequencies are relatively few, and not necessarily appropriate for risk assessment (Simkó, 2019; Leszczynski, 2020; Wood, et al., 2021).

A recent extensive review of the bioeffects literature noted the paucity of studies at high-band $5 \mathrm{G}$ NR frequencies, but concluded: "at the present time, the data are not sufficient to conclude on the existence or not of health effects related to exposure to electromagnetic fields in the band of frequencies around $26 \mathrm{GHz}$ " (ANSES, 2021). The review suggested a long list of needed studies at high band $5 \mathrm{G}$ NR frequencies, in part reflecting the lack of available studies, and in part because of open scientific questions raised by some of the existing studies.

This Perspective focuses on laboratory bioeffects studies, which are relevant to hazard identification, without regard to whether the bioeffects are considered to be thermal or nonthermal in mechanism. Similar considerations might apply to other aspects of the risk assessment process, such as exposure assessment, or to epidemiology as opposed to laboratory studies, but are not presently considered.

\section{RADIO-FREQUENCY ELECTROMAGNETIC FIELDS RISK ASSESSMENT IS DEPENDENT ON STUDY QUALITY}

A health risk exists if a dangerous agent (hazard) is present at a sufficiently high dose (exposure). Both entities thus need to be present simultaneously. Accordingly, a risk assessment (RA) needs to include both exposure assessment and hazard identification.

The primary step in RA is to determine whether a substance in principle can pose a hazard, without taking the dose into account. A health risk for humans can be quantified only if it is known at what dose or exposure level negative effects can be expected. Accordingly, an exposure assessment/characterization must be carried out to determine the actual exposure/dose. If individuals exposed to a potentially hazardous agent at a level below which negative health impacts were determined, the risk may be very low or non-existent even if negative effects are established at higher doses.

The studies presently considered are, for the most part, basic in orientation (as opposed to studies that directly assess adverse outcomes in animals or humans). Such studies may have only indirect bearing on human health risks, but they can clarify the modes of action of potentially hazardous agents, which underpins the RA process, and be valuable in hazard identification.

This Perspective is an extension of a companion Perspective in the same issue (Foster and Vijayalaxmi, 2021) which describes quality issues in one subset of studies involving RF radiation exposures above $6 \mathrm{GHz}$, involving assays of RF-induced genetic damage.

\subsection{Lessons Learned From Main-Stream Toxicology and Nano-Toxicology Studies}

The tenet of toxicology is to identify adverse effects of chemical, physical, or biological agents on living organisms and the environment. This includes to identify the adverse effects' dependency on the exposure routes and on the dose (duration and concentration/exposure). Identification of the dose of a substance that causes toxic effects requires both acute and chronic studies, using standard test procedures.

Nano-toxicology is a relatively new (approx. 15 years) multidisciplinary research area that investigates the interactions of engineered nanomaterials with biological systems. Nanomaterials have unique properties compared with their larger counterparts because of their quantum size effects and large surface area to volume ratio. Therefore a specific emphasis is placed by toxicologists on the correlation between the physicochemical properties of the nanomaterial and effects on the living matter. Initially, toxicologists struggled with all of the unknowns in tests and assays using these new materials, since the interactions with biomolecules significantly change the physicochemical properties of the nanomaterial and thus its toxicity. However, nano-toxicologists identified these primary problems relatively quickly and introduced standard operating procedures (SOPs) for both exposure assessments and biological studies by either adjusting already existing or developing new protocols for standard tests (see e.g., Gottardo et al. (2017)). Nano-toxicology uses good laboratory practice (GLP) with appropriate equipment and OECD guidelines for in vivo and in vitro tests (https://www.oecd.org/env/ehs/testing/section4health-effects.htm effects_20745788). Adoption of these procedures has led to results for certain nanomaterials that are already being used for the European Chemicals Agency's REACH (Registration, Evaluation, Authorisation and Restriction of Chemicals; https://ec.europa.eu/environment/chemicals/reach/ reach_en.htm) initiative.

EMF research regarding possible health effects on the other hand does not adhere to specific guidelines and there are no standardized protocols for either the biological or for the exposure part of the studies. In particular, most if not all, RF bioeffects studies are not done under GLP, many have obvious 
design weaknesses and use statistical methodology that is known to be prone to false discovery (nonreplicable results). [For discussion of genetic toxicity studies see Foster and Vijayalaxmi (2021)].

\subsection{Quality Assessment: Risk of Bias}

The need to assess quality of research for inclusion in systematic reviews or development of regulations is present in all areas of health research. In developing systematic reviews, expert groups commonly rely on defined sets of criteria to assess methodological strengths and weaknesses of studies and classify studies according to risk of bias (RoB). A U.S. National Research Council report (National Research Council (NRC), 2014) defines "risk of bias" as

"... related to the internal validity of a study and reflects study-design characteristics that can introduce a systematic error (or deviation from the true effect) that might affect the magnitude and even the direction of the apparent effect".

Widely used sets of criteria for assessing RoB include the Cochrane Collaborative RoB tool for randomized clinical trials (Higgins et al., 2011) and a set of nine criteria in the OHAT RoB tool for human and animal studies (Translation Office of Health Assessment, 2015). An expert group on EMF at Aachen University has used this tool to assess methodological limitations in studies on biological effects of weak static magnetic fields (Driessen et al., 2020), static electric fields [(Petri et al., 2017; Schmiedchen et al., 2018) and EMF in the intermediate frequency range $(300 \mathrm{~Hz}-1 \mathrm{MHz})$ (Bodewein et al., 2019). For each of nine OHAT RoB criteria, the Aachen group assigned a "risk of bias" score for each paper ranging from "definitely low risk of bias" to "definitely high risk of bias" and assigned the paper to one of three tiers representing different overall risk of bias. These RoB scores determine the weight a particular study is given in the systematic review, or whether a study will be included at all in the assessment. Presently, roughly $100 \mathrm{RF}$ bioeffects studies have been published involving exposures above $6 \mathrm{GHz}$ through the millimeter wave range over a wide range of exposures, examining multiple endpoints (Simkó, 2019). While few of these studies are standardized risk studies (such as the National Toxicology Program (NTP) carcinogen screening assays (https:/ntp.niehs.nih.gov/whatwestudy/testpgm/cartox/ index.html)], they address the biological effects of RF energy and would be carefully reviewed by health agencies. Unfortunately, many of the RF EMF bioeffects paper presently in the literature have insufficient quality to meet criteria for inclusion by health agencies (SSM, 2018) due to lack of appropriate exposure assessment, sham controls, blinding, or other study-design weaknesses (Simkó et al., 2016; Foster and Vijayalaxmi, 2021). Such papers can present unjustified claims that scare the public.

So far, no systematic reviews have been performed of RF bioeffects studies involving exposures above $6 \mathrm{GHz}$ with a level of rigor similar to those produced by the Aachen group in other frequency ranges. Such reviews would presently be limited because of the small number of studies in this frequency range and theiFr extremely diverse nature.

However, three recent reviews of RF bioeffects studies in this frequency range have appeared. In addition to a more comprehensive review by ANSES (2021), (Simkó, 2019; Vijayalaxmi and Prihoda, 2019; Wood et al., 20F21). These studies scored the papers for RoB using the same set of five criteria, some of which parallel the OHAT criteria:

- Blinded study design.

- Appropriate sham controls.

- Positive controls.

- Adequate dosimetry.

- Adequate temperature control.

Two of these reviews Wood et al. (2021) and Simkó (2019) considered studies on all endpoints. The third Vijayalaxmi and Prihoda (2019) considered genetic damage studies over all RF's, including the higher frequency range presently being considered (see the companion paper in this issue by Foster and Vijayalaxmi for discussion of data above $6 \mathrm{GHz}$ ). Almost none of the studies included in these three reviews met all five criteria. Most of the studies (60-68\%) reported biological effects of some sort or other, over wide ranges of exposure conditions and endpoints. The studies presently available at frequencies above $6 \mathrm{GHz}$ are generally small and exploratory in nature.

\subsection{Quality Assessment: Other Aspects of Study Design}

The RoB criteria apply to obvious factors, such as blinding, which affect the internal validity of a study. The importance of a different set of issues related to conduct and reporting of studies has recently come to widespread attention as a result of the "reproducibility crisis" in science. (For an extensive review see Fidler and Wilcox (2021)).

Two such issues that have special relevance to EMF bioeffects studies are misuse of statistics in analyzing data, and unidentified flexibility in study design.

\subsubsection{Misuse of Statistics}

Most of the reviewed bioeffects studies (Simkó, 2019; Vijayalaxmi and Prihoda, 2019; Wood et al., 2021) based their conclusions about "effects" of exposure on some form of null hypothesis significant testing (NHST), such as Student's $t$ test, using $p<0.05$ as the criterion for statistical significance.

However, NHST is well known to be prone to false discovery, i.e., nonreplicable findings (e.g., Colquhoun, 2014; Wasserstein et al., 2019). Gelman (2016) have discussed these difficulties at length and the problems are sufficiently pervasive and severe that some experts recommend that "[NHST] should be abandoned as the cornerstone of research" (Szucs and Ioannidis 2017).

Among many other problems, NHST is designed to evaluate the consistency of a set of data with the "null hypothesis" (H0), that there is exactly zero difference in the relevant statistical measure between the populations from which the samples (data) were drawn (i.e., exposed vs. control groups in the context of RF bioeffects research). 
H0 might fail for either of two reasons: either a real effect is present, or a systematic error introduces difference between the control and exposed populations. The standard practice in many EMF bioeffects studies is to apply the $t$ test and conclude that a "statistically significant" (or "significant") effect is present if $p<0.05$. Since some level of systematic error might be present in any bioeffects study (even if very small), a sufficiently large study (large " $n$ ") can find statistically significant differences between the control and exposed groups due to residual errors in the experiment. In fact, RF bioeffect studies are often examining small apparent effects (e.g., Foster and Vijayalaxmi, 2021 for genetic damage studies) that can be difficult to separate from possible nonrandom errors.

Moreover, the $p$-value is a measure of the consistency of data with the null hypothesis, not the strength of evidence in support of an alternative hypothesis H1 (e.g., a real effect exists). Thus, "it is impossible to tell from the outcome of a single (published) experiment delivering a statistically significant result whether a true effect exist" (Szucs and Ioannidis, 2017).

Both these considerations point to the unreliability of NHST with $p<0.05$ to establish effects of RF exposure. While "statistical significance" is a relevant consideration, the more important questions are the reliability, magnitude, and biological significance of observed findings.

\subsubsection{Unreliable Results Resulting From Unidentified Flexibility of Analysis}

This refers to the ability of investigators to choose alternate methods of analysis of data, which data to include in final results, how to group experimental subjects, etc. during the course of an experiment. This flexibility, if not disclosed, “....allows anything to be presented as significant" (Simmons et al., 2011). The solution to this problem is to pre-specify hypotheses, experimental methods, and methods of analysis, as well as making data available for independent examination. Such precautions are employed in toxicology studies for regulatory approval, which are performed under Good Laboratory Practices (GLP). However few if any RF bioeffects studies are done under GLP nor were they intended for submission to regulatory agencies as parts of new product/drug approval processes. All of the bioeffects studies reviewed by Simkó (2019), Wood et al., 2021, and Vijayalaxmi and Prihoda (2019) are small and many were exploratory in nature, and many have high RoB. Such studies may open up interesting and fruitful scientific questions, but are prone to false discovery. This, together perhaps with confirmation bias of the investigators (who are looking for interesting results) might help to explain in part the large number of "effects" reported in the roughly 100 bioeffects studies in the frequency range above $6 \mathrm{GHz}$.

\subsection{Guiding Principles for High Quality RF-EMF Studies}

From the above discussion, two general sets of criteria need to be satisfied.

\subsubsection{Risk of Bias Criteria}

The most widely used RoB criteria are those from OHAT tools (Translation Office of Health Assessment, 2015). As adapted to in vitro studies, these are:
- Identical experimental conditions across study groups.

- Confidence in the exposure characterization.

- Confidence in the outcome assessment.

- Randomization exposure level.

- Allocation concealment.

- Blinding of research personnel.

- Attrition/Exclusion rate.

- All measured outcomes reported.

- Other potential threats.

The OHAT document indicates that the most important questions to ask are: "were experimental conditions identical across study groups," "can we be confident of the exposure characterization," "can we be confident of the outcome assessment?"

\subsubsection{Study Design and Reporting Should Minimize Number of Investigator Degrees of Freedom}

A number of approaches are possible, including use of GLP, or using methods developed by the Center for Open Science (https://osf.io). These include a series of measures including publication of hypotheses and methods before a study is begun and other "rigor and transparency initiatives."

Such measures are expensive and time consuming for investigators. But it is inexcusable to encourage more poorquality studies that can alarm the public, and at the same time fail to meet quality standards for inclusion in health agency reviews and well-done systematic reviews. The present need is for well done, adequately supported studies using standard protocols, particularly at $5 \mathrm{G}$ high band frequencies.

\section{DISCUSSION AND CONCLUSION}

Competent authorities will ask for and perform risk assessments regarding the possible health effects related to the coming roll-out of $5 \mathrm{G} \mathrm{NR}$. It is most likely that special scrutiny will be applied to assessments that deal with the high frequency band $(25-39 \mathrm{GHz})$, given the relative novelty of such exposures to the public and high level of public concerns bioeffect.

Ideally, health agencies should sponsor multicenter studies to identify and quantify possible health risks from high-band $5 \mathrm{G} \mathrm{NR}$ technologies, given the deficiencies in the current research literature. However, health agencies have expressed little enthusiasm to support such studies, and a recent careful assessment of the scientific literature related to $5 \mathrm{G} \mathrm{NR}$ transmissions does not raise alarms about possible health risks - even as it points to limitations in the current scientific data (ANSES, 2021).

In the absence of large scale and well-funded studies, health agencies will need to rely on smaller studies by individual laboratories, many done under conditions of insufficient funding and technical support. Such studies should be methodologically strong to provide reliable information to health agencies and regulators, and as far as possible avoid false discovery resulting from weak study designs and statistically naïve interpretations of data. That may be 
addressed by studies that are based as far as possible on SOPs and GLP (such as ones provided by OECD (www.oecd.org/ chemicalsafety/testing) or by FDA. As far as the present authors can determine, few if any present bioeffects studies at 5 G NR frequencies satisfy such criteria.

In the bioeffects literature, the general topics of RoB, false discovery from inappropriate use of NHST and flexibility of methodology and analysis of data, have been little discussed (even as they have become major preoccupations in the metascience movement (Fidler and Wilcox, 2021). This Perspective calls attention to the need to address these concerns for future (and much needed) bioeffects studies at $5 \mathrm{G}$ NR frequencies.

\section{REFERENCES}

ANSES (2021). OPINION of the French Agency for Food, Environmental and Occupational Health and Safety on Population exposure to electromagnetic fields associated with the deployment of $5 \mathrm{G}$ communication technology and the related health effects. Maisons-Alfort.

Bodewein, L., Schmiedchen, K., Dechent, D., Stunder, D., Graefrath, D., Winter, L., et al. (2019). Systematic Review on the Biological Effects of Electric, Magnetic and Electromagnetic fields in the Intermediate Frequency Range ( $300 \mathrm{~Hz}$ to $1 \mathrm{MHz}$ ). Environ. Res. 171 (October 2018), 247-259. doi:10.1016/j.envres.2019.01.015

Colquhoun, D. (2014). An Investigation of the False Discovery Rate and the Misinterpretation of P -values. R. Soc. Open Sci. 1 (3), 140216. doi:10.1098/rsos.140216

Driessen, S., Bodewein, L., Dechent, D., Graefrath, D., Schmiedchen, K., Stunder, D., et al. (2020). Biological and Health-Related Effects of Weak Static Magnetic fields $(\leq 1 \mathrm{mT})$ in Humans and Vertebrates: A Systematic Review. PLoS One 15 (6), e0230038. doi:10.1371/journal.pone.0230038

Fidler, F., and Wilcox, J. (2021). "Reproducibility of Scientific Results," in The Stanford Encyclopedia of Philosophy. Summer 20.

Foster, K. R., and Vijayalaxmi (2021). Needed: More Reliable Bioeffects Studies at High Band 5G Frequencies. Front. Comms. Net 2, 721925. doi:10.3389/ frcmn.2021.721925

Gelman, A. (2016). The Problems with P-Values Are Not Just with P-Values. The Am. Statistician 70, 1-2.

Gottardo, S., Crutzen, H., and Jantunen, P. (2017). NANoREG Framework for the Safety Assessment of Nanomaterials. Luxembourg 60. doi:10.2760/245972

Higgins, J. P. T., Altman, D. G., Gøtzsche, P. C., Peter, J., Moher, D., Oxman, A. D., et al. (2011). "The Cochrane Collaboration's Tool for Assessing Risk of Bias in Randomised Trials. BMJ (Clinical Research Ed.) 343 (October), d5928. doi:10.1136/bmj.d5928

IEEE (2019). IEEE Standard for Safety Levels with Respect to Human Exposure to Electric, Magnetic, and Electromagnetic Fields, $0 \mathrm{~Hz}$ to $300 \mathrm{GHz}$. doi:10.1109/ IEEESTD.2019.8859679

International Commission on Non-Ionizing Radiation Protection (ICNIRP) (2020). Guidelines for Limiting Exposure to Electromagnetic Fields (100 $\mathrm{kHz}$ to $300 \mathrm{GHz}$ ). Health Phys. 118 (5), 483-524. doi:10.1097/ HP. 0000000000001210

Leszczynski, D. (2020). Physiological Effects of Millimeter-Waves on Skin and Skin Cells: An Overview of the To-Date Published Studies. Rev. Environ. Health 35 (4), 493-515. doi:10.1515/reveh-2020-0056

National Research Council, (NRC) (2014). National Research Council Review of EPA's Integrated Risk Information System (IRIS) Process. Washington, DC: National Academies Press.

Petri, A-K., Schmiedchen, K., Stunder, D., Dechent, D., Kraus, T., Bailey, W. H., et al. (2017). Biological Effects of Exposure to Static Electric Fields in Humans and Vertebrates: A Systematic Review. Environ. Health: A Glob. Access Sci. Source 16 (1), 41. doi:10.1186/s12940-017-0248-y

\section{DATA AVAILABILITY STATEMENT}

The original contributions presented in the study are included in the article/Supplementary Material, further inquiries can be directed to the corresponding author.

\section{AUTHOR CONTRIBUTIONS}

The work was developed as a collaboration among all authors. $\mathrm{M}-\mathrm{OM}, \mathrm{MS}$, and $\mathrm{KF}$ were contributing to all stages of the ms development. All authors have read and approved the final manuscript.

Schmiedchen, K., Petri, A-K., Driessen, S., and Bailey, W. H. (2018). Systematic Review of Biological Effects of Exposure to Static Electric Fields. Part II: Invertebrates and Plants. Environ. Res. 160 (January), 60-76. doi:10.1016/j.envres.2017.09.013

Simkó, M., and Mattsson, M-O. (2019). 5G Wireless Communication and Health Effects-A Pragmatic Review Based on Available Studies Regarding 6 to 100 GHz. Int. J. Environ. Res. Public Health 16 (18). doi:10.3390/ijerph16183406

Simkó, M, Remondini, D., Zeni, O., and Rosaria Scarfi, M. (2016). "Quality Matters: Systematic Analysis of Endpoints Related to 'Cellular Life' In Vitro Data of Radiofrequency Electromagnetic Field Exposure. Int. J. Environ. Res. Public Health 13 (7), 701. doi:10.3390/ijerph13070701

Simmons, J. P., Nelson, L. D., and Simonsohn, U. (2011). False-Positive Psychology: Undisclosed Flexibility in Data Collection and Analysis Allows Presenting Anything as Significant. Psychol. Sci. 22 (11), 1359-1366. doi:10.1177/0956797611417632

SSM (2018). "Swedish Radiation Safety Authority," in Recent Research on EMF and Health Risk, Twelfth Report from SSM's Scientific Council on Electromagnetic Fields, 2017 (Stockholm.

Szucs, D., and Ioannidis, J. P. A. (2017). When Null Hypothesis Significance Testing Is Unsuitable for Research: A Reassessment. Front. Hum. Neurosci. 11, 390. doi:10.3389/fnhum. 2017.00390

Translation, Office of Health Assessment (2015). OHAT Risk of Bias Tool for Human and Animals Studies. US DHSS: National Toxicology Program.

Vijayalaxmiand Prihoda, T. J. (2019). Comprehensive Review of Quality of Publications and Meta-Analysis of Genetic Damage in Mammalian Cells Exposed to Non-ionizing Radiofrequency Fields. Radiat. Res. 191 (1), 20-30. doi:10.1667/RR15117.1

Wasserstein, R. L., Allen, L., and Lazar, N. A. (2019). "Moving to a World beyond ' $\mathrm{P}<0.05$. Am. Statistician 73 (Suppl. 1), 1-19. doi:10.1080/00031305.2019.1583913

Wood, A., Mate, R., and Karipidis, K. (2021). Meta-Analysis of In Vitro and In Vivo Studies of the Biological Effects of Low-Level Millimetre Waves. J. Expo. Sci. Environ. Epidemiol. March, 1-8. doi:10.1038/s41370-021-00307-7

Conflict of Interest: Author M-OM and MS were employed by the company SciProof International AB. KRF receives minor research support on an unrelated topic (thermal dosimetry) from an industry group (Mobile and Wireless Forum).

Publisher's Note: All claims expressed in this article are solely those of the authors and do not necessarily represent those of their affiliated organizations, or those of the publisher, the editors and the reviewers. Any product that may be evaluated in this article, or claim that may be made by its manufacturer, is not guaranteed or endorsed by the publisher.

Copyright (c) 2021 Mattsson, Simkó and Foster. This is an open-access article distributed under the terms of the Creative Commons Attribution License (CC $B Y)$. The use, distribution or reproduction in other forums is permitted, provided the original author(s) and the copyright owner(s) are credited and that the original publication in this journal is cited, in accordance with accepted academic practice. No use, distribution or reproduction is permitted which does not comply with these terms. 